\title{
Travelers-led Innovation in Sustainable Urban Mobility Plans
}

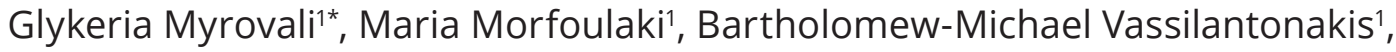 \\ Antonios Mpoutovinas ${ }^{1}$, Kornilia Maria Kotoula ${ }^{1}$ \\ ${ }^{1}$ Hellenic Institute of Transport/Centre for Research and Technology Hellas (HIT/CERTH), \\ 6th km Charilaou-Thermi Road, Thessaloniki, 57001, Greece \\ *Corresponding author, e-mail: myrovali@certh.gr
}

Received: 04 January 2018, Accepted: 31 July 2018, Published online: 15 April 2019

\begin{abstract}
Putting citizens in the position of city planners while giving them a sense of purpose and plans' ownership is a difficult task since the win-win effect of participating in mobility planning is, in the majority of cases, not properly communicated. Aiming to display to travelers the value of their contribution with ultimate scope to increase the participatory approach in Sustainable Urban Mobility Plans (SUMP) concept, the current paper presents the e-platform of MOTIVATE project (MED programme, 2014-2020) developed to cover low citizens' engagement levels. MOTIVATE e-platform is estimated to become a useful tool on the hands of city planners and transport engineers via which, the daunting task of data collection and idea/views/opinions capturing will be facilitated. Furthermore, the exploitation of MOTIVATE e-platform is estimated to support travel behavior change towards environmental friendly ways of transport and increase the acceptability, and thus the efficiency, of sustainable mobility plans.
\end{abstract}

Keywords

sustainable mobility, citizens' engagement, crowdsourcing, decision making support

\section{Introduction}

The growth of urban areas has been constant since the early twentieth century, as a result of a continuous flow of people who choose to move from rural areas in seeking better living conditions. Nowadays, urban areas are formed and inhabited by nearly $55 \%$ of the world's population (percentage that is considered to increase up to $68 \%$ in 2050), and cover an area of just $2 \%$ of the total surface of the planet (United Nations, 2018). A simple insight of these two rates is a strong proof of urbanization's negative side, with immediate impact on people's lives, health, environment (air/water pollution), social relationships (alienation, isolation) and economy (increased cost of life). Particularly in the European Union, the majority of citizens (almost $72 \%, 2007$ data) live in urban environment. To satisfy their daily needs, they all use the same infrastructures and the same transportation system. Urban mobility is a vital part of daily cities' operation, being blamed however for the $40 \%$ of $\mathrm{CO} 2$ emissions and up to the $70 \%$ of the rest pollutants. European cities face increasing problems caused by increasing transport demand levels. Strengthening mobility while reducing congestion and air pollution look like being the key to a sustainable growth path, being simultaneously a great challenge for the cities.
EU's actions towards the formulation of sustainable cities have their origins in the early 90's with the publication of the Green Paper on the Urban Environment that stresses the need for identifying possible lines of actions for the improvement of urban areas. Since then, the European Commission has not stopped to deal with urban areas in general and in particular with urban mobility, fact that proves the great challenges cities face regarding this field. Having regard to the Treaty of Amsterdam (where sustainable development was introduced as an objective for the Community) and the Gothenburg European Council (which established a strategy for sustainable development), the White Paper on Transport Policy of 2001 (European Commission, 2001) make an introduction by stating that a modern transport system should be sustainable from an economic, social as well as environmental viewpoint. In 2006, the mid-term review of the White Paper introduced two important comments; mobility must be detached from its negative side-effects rather than economic activity and the concept of co-modality must become familiar to the passengers so as to achieve modal shift. The Green Paper on Urban Transport (European Commission, 2007) was adopted on 25 September 2007 and intended to start a 
debate on the key issues of urban mobility, with one paramount idea: "To be effective, urban mobility policies need to be based on an approach which is as integrated as possible". Throughout the text, and in line with the approach previously taken in the White Paper, a strong emphasis is given on the exchange of good practice as an instrument that could be promoted at a European level. The Green Paper (European Commission, 2009) concludes by highlighting the need for creating more accessible and secure, greener and smarter (exploiting the advantages of new technologies / Intelligent Transport Systems) cities. Following the Green Paper, EC adopted in 2009 the Action Plan on urban mobility which proposed twenty measures for competent authorities to take in order to promote sustainable urban mobility among which sustainable urban mobility plans development, creation of high quality - affordable public transport, improved reliability, information provision, safe and easy access to all, promotion of lower, cleaner and zero emission vehicles, internalisation of external costs, optimization of existing and exploration of new funding sources and ITS use. Two years after, in 2011, the 2011 Transport White Paper 'Roadmap to a Single European Transport Area' (European Commission, 2011) was published posing as goal for 2050 the exclusion of conventionally-fuelled cars in cities while highlighting that demand management can lower traffic volumes and that facilitating alternative to private car ways should be integral part of cities' agendas. Three of the initiatives pointed at the last White Paper, establishing procedures for SUMPs development - developing road user charging and access restriction schemes and best practice guidelines to better monitor and manage urban freight flows, were then introduced in the Urban Mobility Package of 2013 (European Commission, 2013).

Despite the large policy behind SUMPs and although different aspects of urban mobility management and plans development have been studied in depth by many European projects and initiatives (e.g. ELTISplus, CIVITAS MODERN, CIVITAS ELAN, CIVITAS MIMOSA, PolySUMP, CH4LLENGE, ADVANCE) year now, SUMP's adoption rate in European and especially in MED cities remains notable low. Seeking within reasons potentially blocking the adoption of acceptable and thus effective plans, public rejection and public indifference cannot be overlooked. According to Dobos et Jenei (2013), the actual meaning of engagement corresponds not only to information provision for awareness raising but also - and strongly - to reinforcing the sense of community and building up citizenship. Previous research has indicated major barriers in engaging citizens in transport planning (Bickerstaff and Walker, 2001) which extend from limited awareness and knowledge on the role of citizens and their power in planning to lack of authorities capacity in providing opportunities for participation. From the other side, Booth and Richardson (2001) have highlighted the benefits from citizens' involvement in enhancing planning quality that include enlarged brainstorming, evaluating and testing evidence and addressing uncertainty. Gaventa and Barrett (2012) refer to the engagement as a way of strengthening a sense of citizenship and building responsive states.

The level of understanding of the benefits arising from citizens' participation varies from country to country. As stated in the CH4LLENGE project, there are countries with formal, mandatory procedures (e.g. Germany) for planning activities under a participatory spectrum and countries without these formal procedures. Furthermore, there are countries (e.g. Belgium) that can be seen as donor of experience since having extensive experience in innovative participation instruments in complex planning processes.

The two most frequently used guidelines for SUMP development are the these developed by the EU and these by JASPERS, both of which offer suggestions on how to create a long term vision for sustainable urban mobility (Interreg Europe, "Policy Brief"). In this paper we focus on the EU SUMP guidelines. The thorough study of the widely known "Planning cycle for a sustainable urban mobility plan", as presented in the most EU SUMP guidelines triggered the idea of the MOTIVATE platform development (implemented in the framework of MOTIVATE project (MED 2014-2020 programme)). The platform is an ICT based tool that tries to strengthen citizens' involvement in the mobility planning process. The term ICT based tool for encouraging citizens' involvement in the sustainable planning procedure refer to, free of time and location constraints, technology-mediated forms of citizens' participation; no need for physical attendance at conventional stakeholders events where citizens are usually represented by just few members taking the role of simple observers (Misra et al, 2014; Slotterback et al., 2010; Wagner, 2013; Myrovali et al., 2018).

Focusing on citizens' involvement in the development and implementation of SUMPs, the MOTIVATE app tries to capture citizens' and visitors' mobility habits \& needs and composing the respective trip diaries, developing in this way a good (database) starting point for the authorities to plan interventions and improve infrastructures and services operation provide users (travellers) with 


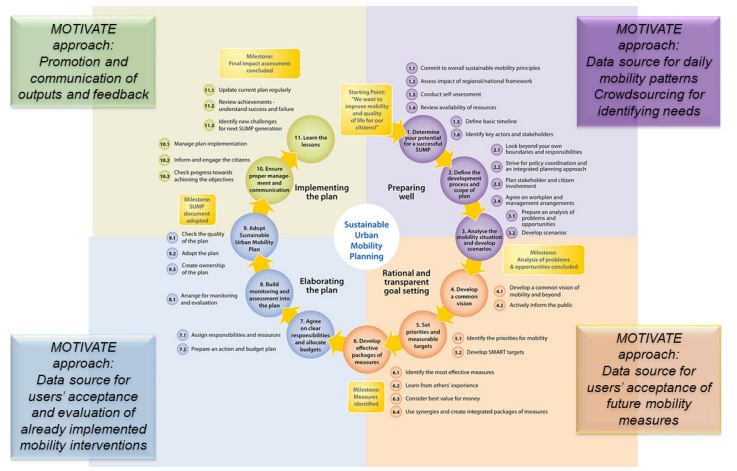

Fig. 1 Core contribution of MOTIVATE's outputs in SUMP's cycle

sustainability related notifications motivating modal shift towards alternative to private car modes promote to the wider public sustainable mobility interventions, achieving high level of awareness raising and involve travellers in a long lasting and effective open dialogue for capturing:

- Dairy trips (O-D matrix)

- opinions \& suggestions on mobility issues (current operation of public transport modes - level of service, occupancy rates, offenses etc, road network conditions, accidents etc)

- acceptance level for potential interventions

In order to "motivate" the travellers to use daily the specific platform offering their personnel data specially regarding their O-D trips, a game was also implemented which gives the opportunity to users not only to create their own sustainable city but also to interact with the above mention services, as they earn points for the game as much as they use the first three services of the platform.

The architecture of the MOTIVATE platform and the operational characteristics of the services and the game are presented analytically in the next sections.

\section{The architecture of the MOTIVATE platform}

\subsection{Components of the platform}

The MOTIVATE Platform consists of multiple elements and integration of several components. Below is an overview of the overall architecture which consists of both front end and backend services and functionalities.

The platform consists of:

- Mobile Apps for mobile devices, Smartphones/ Tablets for at least Android and iOS devices

- Web Portal for access over web browsers

- Web Services for exchanging data

- Data Storage

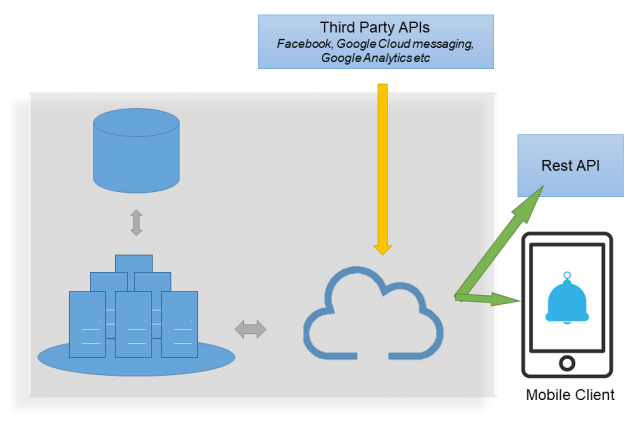

Fig. 2 Basic components of the MOTIVATE platform

The system intends to create an open, globally accessible and complete set of information services able to support the needs of the service. The system is developed with a technical architecture divided into three layers (1) the Presentation layer, (2) the Business layer and (3) the Data layer. The presentation layer is responsible for accepting user input and rendering the user interface that is returned from web services. User Interface using various SDKs such as Android, iOS, .NET, JavaScript, etc depends on the clients platforms (mobile, web). The application layer consists of 2 mobile clients, one for Android and one for iOS with which the user interacts. Also the system has a REST API which can be used to develop a web interface or to communicate with other systems. The business layer implements the core functionality of the system and encapsulates the relevant business logic. The system is structured around the concepts of business processes and business components. Many business processes involve multiple steps that must be performed in the correct order. Business workflow components define and coordinate long running, multistep business processes, and can be implemented using business process management tools.

The data layer provides access to data that is hosted within the boundaries of the system. Data access components in this layer are responsible for exposing the data stored in databases to the business layer. The system requires information from external systems to complete a business process. Therefore, business components must access external services or applications. A service gateway is a component that encapsulates the interface, protocol, and code required to use such services. The service is a Web service that uses REST over HTTP for communications and is fully described by means of Web Services Description Language (WSDL). The service defines a contract that all service consumers must conform in order to access the service. The contract defines such things as the 
technology, communications protocols, and message definitions needed to communicate with the service.

\subsection{Web application architecture}

Motivate Platform services are served to the end user over web for any browser that can interpret HTML content. The development of the web application is achieved using .NET framework with $\mathrm{C} \#$ programming language for the backend procedures while for the UI and the client-side HTML, CSS, JavaScript, jQuery and AJAX. The data interoperability between the backend and the web front end application is achieved with RESTful services over the Parse-Server.

The Motivate Web application is built on responsive techniques so can be easily accessible and readable by different browsers, window sizes and devices. The core layout of the web platform is based on a master page which provides a common appearance and behavior to all sections of the platforms while allowing an update to the site with minimum effort. Also, several sections of the platform are built as user controls, instead of pages, so this makes the relative content and/or service reusable at least within the portal. The master page and the pages is developed using Cascading Style Sheets (CSS) for the layout with HTML while the content is rendered through the web services over AJAX. Pages and user controls are mostly developed using client-side scripts such as JavaScript and jQuery in order to improve the users experience and to provide better responsiveness. The navigation of the web platform is made with the usage of a top main menu as well as required link whenever is necessary to provide the user with the relative information.

\subsection{Mobile application architecture}

The provided services by MOTIVATE Platform are running on the mobile devices running (Android or iOS). The development of the mobile apps is achieved with the usage of the Parse-server and other native tools for developing mobile apps depending on the platform. For iOS development Parse-server API provides all the necessary functionalities to gather and submit data while Swift programming language is used for the development of the UI and the internal functionalities of the Motivate client iOS devices. For Android development Parse-server API provides all the necessary functionalities to gather and submit data while Java programming language is used for the development of the UI and the internal functionalities of the Motivate client Android devices. In both cases the client Apps supports a variety of different device hardware (ex. Different screen sizes) and software (ex. Lollipop or higher). Mobile

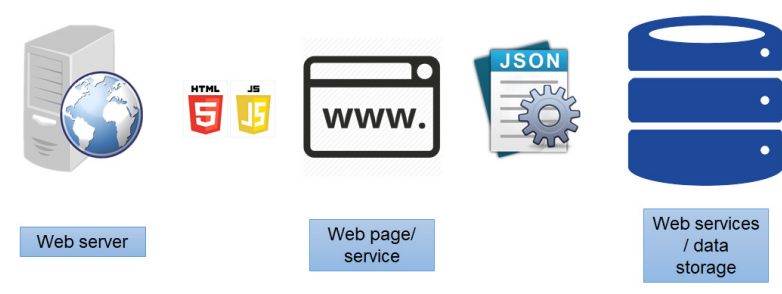

Fig. 3 Service Oriented single page web application

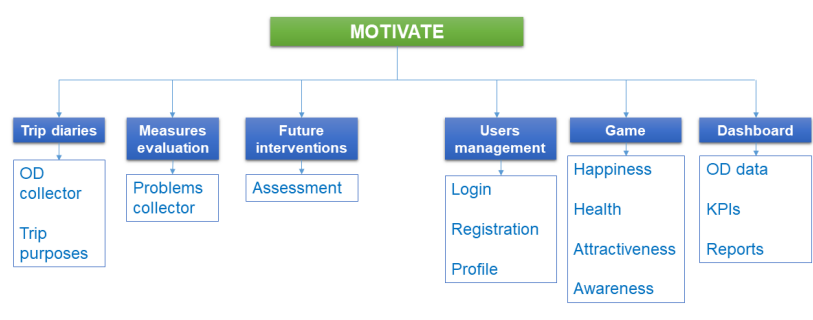

Fig. 4 Functional decomposition of the Platform

device design and development is unique due to the constrained and differing nature of device hardware. Targeting multiple devices with very different hardware parameters. The heterogeneous device environment is something that was kept in mind when designing Motivate mobile application. Factors include variations in screen size and orientation, limitations in memory and storage space, and network bandwidth and connectivity. The following were respected:

- Optimize the application for the device by considering factors such as screen size and orientation, network bandwidth, memory storage space, processor performance, and other hardware capabilities.

- Consider device-specific capabilities that you can use to enhance your application functionality, such as accelerometers, graphics processing units (GPUs), global positioning systems (GPS), haptic (touch, force and vibration) feedback, compass, camera, and fingerprint readers.

\section{Core platform's functionalities}

The MOTIVATE platform consists of the following functional areas. Each functional area represents either a different service to the end users or a functionality to support the services.

\subsection{Trip Diaries}

The Trip Diaries section collects the user's daily trip. The users has either to setup some of their personal information, such as gender, age group, working status, educational level, city etc. or the system fills the appropriate fields based on the registered user profile info. The user, 


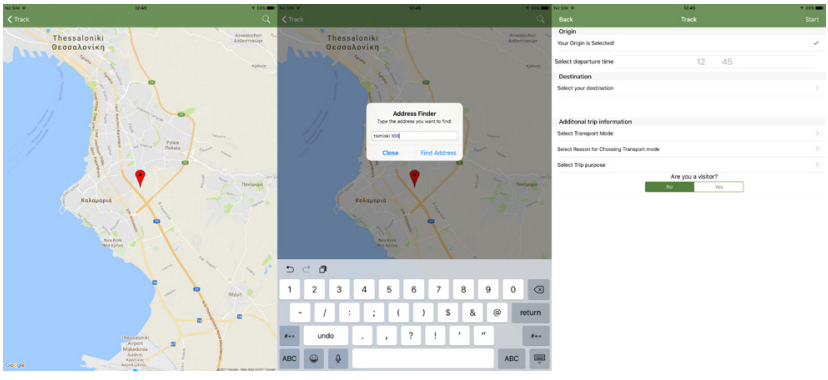

Fig. 5 Screenshot of the MOTIVATE trip diaries application

also, must set up his/her origin and destination, the transport mode and the trip purpose as well as the reason for choosing the desire transport mode.

Once a trip is completed, the system stores the relative trip data, such as trip start and end time (and date) stamp, the GPS data like latitude, longitude, speed etc. the overall travel time and the average trip speed and all this in relation to the user selected data.

At the end of each trip recording, the system also calculates relative awarded points based on the user's selected input and adds them to the Motivate game (4th functionality) for the user to expand his/her game city and make it further sustainable.

\subsection{Measures Evaluation}

The Measures Evaluation service provides the end users (citizens \& tourists) the opportunity to evaluate existing city status and conditions. The users select the city and the measure to evaluate and evaluate specific measure-related criteria using a scale from 1 to 7 .

Once an evaluation is submitted the system stores the relative ratings in relation to the user selected data. Points per evaluation are earned for playing the MOTIVATE game.

\subsection{Future Interventions Assessment}

The Future Interventions Assessment service provides the end users (citizens \& tourists) the ability to participate (in a way) to the city's future policies, expansion and measures by assessing possible interventions that may apply to their city. The user states its perception as for the contribution of a planned intervention in certain areas (necessity is assessed in the current service). As in the previous functionalities, the user earns points per each assessment.

\subsection{Game}

The purpose of the game is to make the users familiar to sustainability, how it can be achieved and how everyday decisions that they make can affect the entire urban

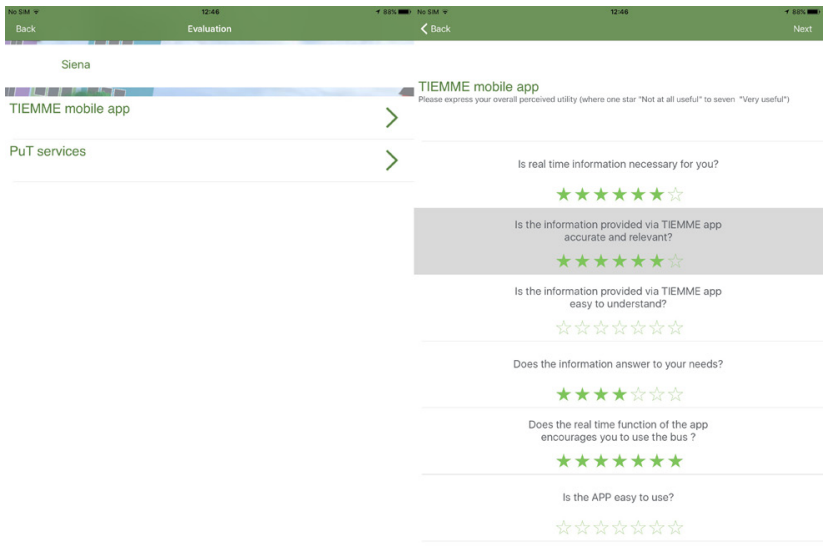

Fig. 6 Screenshot of the MOTIVATE measures' evaluation application

environment both in the short- and medium-term. To make the game even more closer to real life, it is a simulation of an urban environment reflecting the citizens mobility in the city. In more detail, the environment into residential and commercial, where people live and work respectively. Furthermore, in the environment there are three available means of transportation:

- Walking/cycling through the city's pedestrians' network (or walking routes) or bicycling network

- Public transportation in the form of buses or trams

- And the use of private cars

- Each citizen must make the following decisions:

- If his/her movement goal is to get at home or at work

- If the appropriate amount of time has passed, in which the citizen must stay at work or at home

- The means that will use for transportation

- Perform the movement

Each citizen's decision changes several characteristics of the environment: for example how many private cars circulate, how many means of public transportation are necessary (or if the current ones are adequate) and the pollution level in the form of $\mathrm{CO} 2$ emissions. Moreover, the urban environment has two other characteristics that are affected by individual choices; however they are not easy to quantify. These are the aggregate "happiness level" and the health level of the city.

These two characteristics are Key Performance Indicators that summarize in a meaningful way the "happiness" and health level of each citizen. The change of these environment characteristics in turn affects the individual characteristics and actions of each citizen ("happiness" and health levels, and the choice of transportation). Thus, a loop is formed where the characteristics and 
actions of each individual citizen affects the characteristics of the entire group of citizens, which in turn affects the characteristics of the environment, which finally affects back both the individual and collective characteristics, and actions of the citizens.

Thus, for the concept of the game does not involve the user/player; all the interactions, the updates and the movements are performed automatically. However, for it to be a game it must involve the user. As a result, the user can act on the game on a top-down approach as an entity with power over several characteristics of the game. These interventions/game actions are:

- On the urban environment, where the player can act upon the availability of public means of transportation and the routes for walking/cycling

- On the aggregate citizens' characteristics, where the player can act/manipulate their choice of transportation (it can be thought as campaigns that urge citizens to avoid the use of private cars)

- These two game actions have a dual purpose which describe the objective of the game:

- Each player must try to achieve the highest possible levels of "happiness" and health levels before the end of the game cycle.

- Each player must avoid the aggregate "happiness" and health levels to become zero.

The game itself has also as an objective to inform the players about the complexity of the transportation systems in the context of an urban environment and how an individual choice can affect the entire urban system (Myrovali et al., 2018).

\section{Discussion}

Travelers-led innovation is what today's sustainable communities urgently asks for; active travelers providing constant feedback to transportation planners can undoubtedly add value to fit-to-real needs decision making process. However, although travelers' contribution is of major importance for developing effective and acceptable mobility plans, their engagement is still one of the most challenging tasks to be overcome by local authorities. The current work presented the MOTIVATE platform, an ICT based tool enabling citizens' participation in mobility planning. The main benefit provided through the platform is the very close interaction between the travelers (citizens and visitors) inside a city and the transport "decision makers". The travelers become the central source of data and information while the policy makers and the stakeholders are planning upon their needs as graphically presented in Fig. 7.

The MOTIVATE platform core actors and simultaneously the beneficiaries are:

- Travelers: The end users (citizens, commuters, visitors etc) provide useful data that will be used for the implementation of cities' SUMPs and the upgrade of the transport system operation. In particular by declaring their everyday travel patterns, the authorities receive a clear picture on the main traffic poles in the city (generators and attractors). Furthermore, stating their satisfaction from existing measures and their perception (necessity) for future mobility interventions they assist decision making. From the other side, they become active parts of their community and accept the satisfaction of being part of measures selection and implementation. As a result, they respect the mobility plans and become recipients of a well-functioning, sustainable environment.

- Transport providers: Transport providers, such as public transport organizations, taxi unions, interurban bus companies, provide to the platform data concerning their transport services or operational characteristics that should be evaluated or assessed by the commuters. On the other hand, the platform makes available to these providers, data from the use of the apps, such as residents and visitors O-D patterns as well as the results of their assessment about the transport services and recommendations. The latter helps them improve both their business models and their market share.

- City policy makers: Beyond the end users, the city policy makers are the main beneficial of the platform as the platform provides useful data that will be used for the implementation of the city SUMP. In particular, the platform makes important contribution with respect

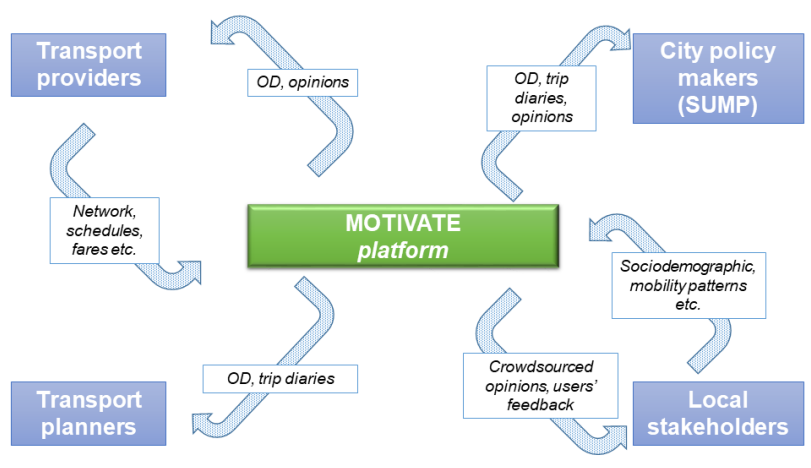

Fig. 7 Interaction diagram of the MOTIVATE platform 
to the use of the local transport system, O-D patterns, trips diaries, and opinions that would make the local mobility system more attractive and sustainable.

- Local stakeholders: Local stakeholders, such as commercial associations, chambers, hotels, tour operators, travel agencies, schools, NGOs and other local associations, can provide their knowledge about the needs of travellers and residents, in order to decide the transport services or operational characteristics that should be evaluated or assessed by the commuters. On the other hand, the platform could send to these stakeholders the assessment and opinions of the users about the available mobility services.

- Transport planners: Transport planners will also benefit from the platform, as they can obtain O-D patterns and trips diaries that can help them in their transport studies and planning of the local transport infrastructures.

\section{References}

Bickerstaff, K., Walker, G. (2001) "Participatory Local Governance and Transport Planning", Environment and Planning A: Economy and Space, 33(3), pp. 431-451. https://doi.org/10.1068/a33173

Booth, C., Richardson, T. (2001) "Placing the Public in Integrated Transport Planning", Transport Policy, 8(2), pp. 141-149. https://doi.org/10.1016/S0967-070X(01)00004-X

Böhler-Baedeker, S., Lindenau, M. (2013) "Why is Participation a challenge in sustainable urban mobility planning?" CH4LLENGE project, [pdf] Intelligent Energy Europe Programme of the European Union, Available at: http://www.eltis.org/sites/ default/files/trainingmaterials/challenge_participation_final.pdf [Accessed: 03 January 2018]

European Commission (2001) "White Paper - European transport policy for 2010: time to decide", Brussels, (370 final).

European Commission (2007) "Green Paper - Towards a new culture for urban mobility", Brussels, (551 final),

European Commission (2009) "Communication from the Commission to the European Parliament, the Council, the European Economic and Social Committee and the Committee of the Regions - Action Plan on Urban Mobility", Brussels, (490 final).

European Commission (2011) "WHITE PAPER: Roadmap to a Single European Transport Area - Towards a competitive and resource efficient transport system", Brussels, (144 final).

European Commission (2013) "Communication From the Commission to the European Parliament, The Council, The European Economic and Social Committee and the Committee of the Regions: Together towards competitive and resource-efficient urban mobility", Brussels, (913 final).

Dobos, A., Jenei, A. (2013) "Citizen Engagement as a Learning Experience", Procedia - Social and Behavioral Sciences, 93, pp. 1085-1089

https://doi.org/10.1016/j.sbspro.2013.09.335
- Summing up, the benefits of the platform refer to the community as a whole since both the decision making process is enhanced due to the increased citizens participation and the travelers will experience a community serving real needs. Future enlargement of the cities participating in the MOTIVATE e-platform are already planned as well as future content enhancement so as to provide further input for mobility planning. An embedded version of services and the game is also under consideration.

\section{Acknowledgements}

The MOTIVATE app and platform was developed by CERTH/HIT in the framework of MOTIVATE project, "Promoting citizens' active involvement in the development of Sustainable Travel Plans in Med Cities with Seasonal Demand" - MED Programme 2014-2020, co-financed by the European Regional Development Fund.

Gaventa, J., Barrett, G. (2012) "Mapping the Outcomes of Citizen Engagement", World Development, 40(12), pp. 2399-2410. https://doi.org/10.1016/j.worlddev.2012.05.014

Misra, A., Gooze, A., Watkins, K., Asad, M., Le Dantec, C.A. (2014) "Crowdsourcing and Its Application to Transportation Data Collection and Management", Transportation Research Record, 2414(1), pp. 1-8.

https://doi.org/10.3141/2414-01

CERTH/HIT (2017) "D3.2.1 / Design of the Logical Architecture of MOTIVATE platform" [online] Available at: https://motivate.interreg-med.eu/fileadmin/user_upload/Sites/Urban_Transports/Projects/ MOTIVATE/D3.2.1_Design_of_the_Logical_Architecture of_MOTIVATE_platform.pdf [Accessed: 03 January 2018]

Myrovali, G., Tsaples, G., Morfoulaki, M., Aifadopoulou, G., Papathanasiou, J. (2018) "An Interactive Learning Environment Based on System Dynamics Methodology for Sustainable Mobility Challenges Communication \& Citizens' Engagement", In: Dargam, F., Delias, P., Linden, I., Mareschal, B. (eds.) Decision Support Systems VIII: Sustainable Data-Driven and Evidence-Based Decision Support. ICDSST 2018, Lecture Notes in Business Information Processing, Vol 313, Springer, Cham, Switzerland, pp. 88-99.

https://doi.org/10.1007/978-3-319-90315-6_8

Slotterback, C. (2010) "Public Involevment in transportation project planning and design", Journal of Architectural and Planning Research, 27(2), pp. 144-162.

United Nations, Department of Economic and Social Affairs, Population Division (2018) "World Urbanization Prospects: The 2018 Revision, Methodology", United Nations, New York, (ESA/P/WP.252).

Wagner, J. (2013) "Measuring the Performance of Public Engagement in Transportation Planning: Three Best Principles", Transportation Research Record, 2397(1), pp. 38-44. https://doi.org/10.3141/2397-05 